\title{
Validación de una Escala sobre Work Engagement. Perfiles asociados a alta Performance y Satisfacción Laboral
}

\author{
Validation of a Work Engagement Scale. Profiles associated with high Performance and Job \\ Satisfaction
}

\author{
María Laura Lupano Perugini ${ }^{1}$ \\ Guadalupe de Ia Iglesia ${ }^{2}$ \\ Alejandro Castro Solano ${ }^{3}$ \\ Mercedes Fernández Liporace ${ }^{4}$
}

\author{
1,2,3,4 Facultad de Psicología, Universidad de Buenos Aires. Argentina \\ 1,2,3 Facultad de Ciencias Sociales, Universidad de Palermo. Argentina \\ ${ }^{1,2,3,4}$ Consejo Nacional de Investigaciones Científicas y Técnicas. Argentina
}

\begin{abstract}
Resumen: El objetivo principal de este estudio consistía en la validación de una escala para la evaluación del work engagement -compromiso con el trabajo-, considerado como una presencia psicológica en el rol que incluye atención, absorción y energía dirigida a tareas laborales. Mediante análisis factorial confirmatorio, estimación de la consistencia interna y evidencias de validez convergente y discriminante, se confirma que la Escala Argentina de Compromiso con el Trabajo es una prueba válida y confiable para su uso en población argentina. Además, se buscó analizar si existen diferencias en los niveles de work engagement de acuerdo con variables individuales y organizacionales. Se observaron mayores niveles de atención y absorción en sujetos de menor edad y menores niveles de atención en empleados con personal a cargo. Por último, se identificaron perfiles de engagement asociados a alta performance y satisfacción laboral.
\end{abstract}

Palabras clave: engagement, satisfacción laboral, performance, psicología laboral, psicología organizacional

\begin{abstract}
The aim of this paper was to validate a scale to assess work engagement: a measure of a psychological aspect of the role that includes the attention, absorption and energy that the individual invests in his/her work tasks. Confirmatory factor analysis, internal consistency estimation, and evidences of convergent and discriminant validity indicated that the Argentine Work Engagement Scale is a valid and reliable measure to be used in Argentinean population. Additionally, differences in work engagement levels were studied regarding individual and organizational variables. Higher levels of work engagement were found in younger individuals and in those who were in charge of personal. Lastly, some engagement profiles were found to be associated to higher performance and job satisfaction.
\end{abstract}

Key Words: engagement, work satisfaction, performance, work psychology, organizational psychology

Cómo citar este artículo:

Lupano Perugini, M. L., de la Iglesia, G., Castro Solano, A., \& Fernández Liporace, M. (2017). Validación de una Escala sobre Work Engagement. Perfiles asociados a alta Performance y Satisfacción Laboral. Ciencias Psicológicas, 11(2), 127-137. doi: https://doi.org/10.22235/cp.v11i2.1482

Este estudio recibió subsidio de los siguientes proyectos: PIP CONICET 11220150100381CO "Hacia una aproximación émica de la psicología positiva. Los rasgos positivos como predictores del funcionamiento óptimo" y Proyecto UBACyT, 20020150100037BA "La evaluación de los rasgos de personalidad positivos. Su relación con los rasgos de personalidad patológicos (DSM5) y el bienestar psicológico”. (Director: Alejandro Castro Solano)

Correspondencia: María Laura Lupano Perugini, Av. Dorrego 1279 (CP: C1414CKT). CABA, Argentina, e-mail: mllupano@hotmail. com; Guadalupe de la Iglesia, e-mail: gdelaiglesia@gmail.com; Alejandro Castro Solano, e-mail: alejandro.castrosolano@gmail. com; Mercedes Fernández Liporace, e-mail: mliporac@psi.uba.ar. CONICET. 
El objetivo principal de este estudio reside en la validación de una escala destinada a la evaluación del work engagement -compromiso con el trabajo-. En los últimos años ha crecido el interés por estudiar este tema puesto que se ha verificado su papel central en la obtención de buenos resultados tanto para el empleado como para la organización en su conjunto (Rothbard \& Patil, 2012).

Desde la Psicología Organizacional Positiva (POP) -iniciada por Dutton, Cameron y Quinn (Cameron \& Spreitzer, 2012)- se propone el análisis de fenómenos como el engagement, la satisfacción laboral o el compromiso de los miembros con las organizaciones, temáticas que antes no habían sido abordadas debidamente por el énfasis puesto en el estudio de los aspectos negativos y sus consecuencias para las organizaciones (Lupano Perugini, 2014).

La definición de work engagement ha sido muy discutida en el campo de la POP (Bakker, Schaufeli, \& Leiter, 2008). Gran cantidad de autores coinciden en señalar inconsistencias tanto en las diferentes definiciones del concepto como en los instrumentos desarrollados para su medición (e.g. Christian, Garza, \& Slaughter, 2011; Hirschfeld \& Thomas, 2008; Jeung, 2011; Macey \& Schneider, 2008; Masson, Royal, Agnew, \& Fine, 2008; Nienaber \& Martins, 2014; Robertson \& Cooper, 2010; Rothbard \& Patil, 2012; Schaufeli \& Salanova, 2011; Viljevac, Cooper-Thomas, \& Saks, 2012). Tal variedad de definiciones redunda en estudios cuyos resultados no son pasibles de comparación y generalización (Nienaber \& Martins, 2014; Vilijevak et al., 2012).

La mayor parte de los investigadores citados consideran que el work engagement es un constructo multidimensional que puede confundirse con otros constructos relacionados (e.g. compromiso organizacional, satisfacción laboral, identificación con el trabajo) pero la falta de consenso aparece a la hora de definir las dimensiones que lo conforman. El presente estudio adopta la conceptualización propuesta por Rothbard y Patil (2012), que reúne los antecedentes más significativos sobre el término. Estos autores sostienen que se trata de una variable de nivel individual (no organizacional) y la definen como "una presencia psicológica en el rol -'estar ahí'-. Incluye la atención, absorción y energía de la persona dirigida hacia las tareas laborales" (p. 59). Por lo tanto, tienen en cuenta dimensiones tales como la atención -que refiere a los recursos motivacionales que una persona puede aplicar a una tarea determinada-, la absorción-que refiere a la capacidad de aplicar esos recursos con intensidad- y la energía- que consiste en un componente físico que puede ser dirigido a la tarea-. Atención y absorción representan los subcomponentes cognitivos del constructo y energía, el subcomponente físico (Rothbard, 2001).

En relación con los antecedentes más relevantes del término, las investigaciones de Kahn (1990, 1992) sirvieron de base para su caracterización como un estado que denota por la presencia psicológica del individuo en su rol laboral. Posteriormente, diversos estudios han analizado la composición del constructo. Por ejemplo, Rothbard (2001) fue el primero en sugerir las dimensiones atención y absorción. Otros antecedentes significativos se encuentran en los trabajos de Maslach, Schaufeli y Leiter (2001), que definieron el work engagement como un constructo opuesto al burnout, ambos situados en un continuum que va desde el polo negativo del cansancio, el desgaste, el cinismo y la despersonalización en el trabajo, hacia la energía, el compromiso, el involucramiento y el sentido de pertenencia. Posteriormente, Schaufeli y Bakker (2004) efectuaron una revisión de este enfoque ya que, desde su punto de vista, el opuesto de burnout no necesariamente implicaba la noción de engagement. De este modo propusieron tres dimensiones que formarían parte del constructo: vigor-que refiere a altos niveles de energía mental y resiliencia-, dedicación - que remite a ser desafiado e inspirado por el trabajo - y absorción - que consiste en concentrarse totalmente en el propio trabajo. Muchos trabajos han utilizado este abordaje, pero otros han criticado su dependencia teórica respecto del burnout, indicando que algunas de las dimensiones propuestas por Schaufeli y Bakker se solapan con otros constructos -e.g. afecto positivo- (Zhang, Rich, \& LePine, 2009). Rich y otros colegas retomaron la conceptualización original de Kahn $(1990,1992)$ y las dimensiones propuestas por Rothbard (2001), proponiendo una definición que cuenta con tres componentes, uno físico -que alude a la energía física puesta en el trabajo-, uno emocional -que involucra una sensación placentera y la activación de afecto positivo- y uno cognitivo - que implica la absorción y la atención(Rich, LePine, \& Crawford, 2010). Esta definición también presenta algunas dificultades, como el solapamiento del componente emocional con el constructo afecto positivo, o el hecho de reunir absorción y atención en una única dimensión, 
en tanto que la evidencia sugiere que se trata de dimensiones separadas (Rothbard, 2001).

En virtud de las dificultades expuestas en los antecedentes citados, se escoge para el presente trabajo la definición propuesta en párrafos previos de Rothbard y Patil (2012) que considera dos dimensiones cognitivas (atención y absorción) y una física (energía).

Nienaber y Martins (2014) señalan que, a pesar de las diferencias señaladas en cuanto a su definición, se han diseñado varios instrumentos para medir work engagement, ya sea en el campo académico o aplicado. El más empleado internacionalmente suele ser la Utrecht Work Engagement Scale (UWES), basada en la propuesta de Schaufeli y Bakker (2004). Tiene en cuenta las dimensiones vigor, dedicación y absorción. Sin embargo, muchos han sido los señalamientos en cuanto al constructo en que se basa la escala, así como en relación con sus índices de fiabilidad que, en muchos casos fueron inferiores a lo esperado (e.g. Rothman \& Rothman, 2010). Además los resultados de los estudios factoriales realizados no han logrado replicar las dimensiones del modelo teórico, particularmente en Latinoamérica (Rodríguez-Montalbán; Martínez-Lugo, \& Sánchez-Cardona, 2014). Con base en lo anterior se decide para este trabajo diseñar una nueva prueba a partir de una serie de ítems propuestos por diferentes autores (Rothbard, 2001; Rothbard \& Patil, 2012), que contemplan la definición utilizada en esta investigación. Adicionalmente, las pruebas destinadas a la medición de engagement suelen ser muy breves (la versión actual de la UWES presenta solo 9 ítems), lo que dificulta los procesos de adaptación.

El engagement cobra especial relevancia en el ámbito laboral dada su asociación con variables de resultado, por lo que numerosas investigaciones lo relacionan tanto con altos índices de satisfacción como de performance laboral. Por ejemplo, muchos estudios han encontrado que está fuertemente asociado a la satisfacción laboral dando cuenta de que el estado psicológico de presencia en un rol facilita la sensación de bienestar en el trabajo (e.g. Alarcon \& Edwards, 2011; Høigaard, Giske, \& Sundsli, 2012; Mache, Vitzthum, Klapp, \& Danzer, 2014; Karatepe \& Karadas, 2015). La satisfacción laboral es una actitud que las personas tienen hacia su trabajo y que engloba diferentes facetas (e.g. satisfacción con el supervisor, con los compañeros de trabajo, con la remuneración, con las posibilidades de ascender, así como con el trabajo en general) (Cameron \& Spreitzer, 2012; Spector, 1997). Por otro lado, múltiples estudios han encontrado asociación entre el work engagement y la performance laboral que alude a cuán bien se desenvuelve un individuo en las tareas necesarias para un rol laboral y a cómo contribuye positivamente al contexto social y psicológico de una organización (Christian, Garza, \& Slaughter, 2011). De acuerdo con Christian et al. (2011), un empleado con alto engagement estaría motivado para llevar las tareas de su rol con más persistencia, intensidad y concentración. En relación con lo antedicho, Albrecht, Bakker, Gruman, Macey y Saks (2015) argumentan que la política de Recursos Humanos debe fomentar los niveles de engagement ya que esto repercute en los resultados de la organización, sobre todo si se trabaja en los aspectos vinculados a la energía (Owens, Baker, Sumpter, \& Cameron, 2016). Lin et al. (2016) desarrollaron un estudio en el que verificaron que es crucial intervenir sobre el liderazgo de los supervisores para lograr tales efectos.

Por último, en relación con variables individuales y organizacionales, en algunas investigaciones se ha hallado que los niveles de engagement presentan diferencias según sexo, mostrando las mujeres índices levemente más bajos que los hombres (e.g. Liu, Cho, \& Putra, 2017; Mastenbroek et al., 2014). En cuanto a la edad, los resultados suelen ser contradictorios ya que algunos muestran un incremento (e.g. Goštautaitė \& Bučiūnienè, 2015; Schaufeli \& Bakker, 2004) y otros una tendencia a la disminución de los niveles de engagement según edad (e.g. Avery, McKay, \& Wilson, 2007). Además, se ha verificado que el tipo de tarea al que se dedica el empleado puede influir en sus niveles de engagement. Por ejemplo, quienes realizan tareas docentes suelen reportar menores niveles de vigor y energía (Innstrand, 2016). En este sentido, un trabajo reciente realizado en empresas argentinas informó que en las organizaciones públicas y de tamaño grande se observan menores niveles de satisfacción y de prácticas positivas (e.g. respeto, apoyo, etc.) (Lupano Perugini \& Castro Solano, 2017).

\section{Objetivos}

- Validar una escala para la evaluación del work engagement (compromiso con el trabajo) en sus tres dimensiones (atención, absorción, energía). 
- Analizar diferencias individuales en el nivel de engagement según variables individuales (e.g. sexo, edad, personal a cargo) y organizacionales(e.g. tamaño, tipo de empresa).

- Identificar perfiles de engagement asociados a alta performance y satisfacción laboral.

\section{Método}

\section{Participantes}

Se empleó una muestra voluntaria de 569 empleados (288 hombres, 50.6\%; 281 mujeres, $49.4 \%$ ) con una edad promedio de 36.85 años $(D E=11,7)$. La mayoría residía en Ciudad de Buenos Aires, CABA-y provincia de Buenos Aires $(96.8 \%, n=545)$, el 3.9\% $(n=22)$ en provincias del interior de Argentina y solamente el $0.4 \%$ $(n=2)$ vivía transitoriamente en el exterior.

En relación con variables organizacionales, el 81.9\% $(n=466)$ de los empleados pertenecía a empresas privadas mientras que el 18.1\% $(n=$ 103) se desempeñaba en empresas públicas. En cuanto al tamaño de la empresa, el 42.4\% $(n=241)$ pertenecía a grandes empresas, el 33.9\% $(n=193)$ a medianas y el $23.7 \%$ a pequeñas $(n=135)$. Del total de participantes solo el $33.9 \%(n=193)$ tenía personal a cargo, en tanto que el resto ocupaba una posición de subordinado $(66.1 \%, n=376)$.

\section{Materiales}

- Escala Argentina de Compromiso con el Trabajo -EACT-: Se trata de una prueba especialmente diseñada para la evaluación del work engagement (compromiso con el trabajo) con base en la propuesta teórica de Rothbard y Patil (2012), que se refiere a un constructo multidimensional vinculado a la dedicación de un empleado a su rol y tareas laborales; está compuesto por dos dimensiones cognitivas (atención y absorción) y una física (energía). Como resultado del proceso de validación se obtuvo una versión final de 11 ítems (e.g. "Cuando estoy trabajando, a menudo pierdo la noción del tiempo”) con respuesta de formato Likert de cinco puntos, con recorrido de 1 (completamente en desacuerdo) a 5 (totalmente de acuerdo). Mediante análisis factorial confirmatorio se pudo verificar tal estructura de tres factores. Además, la consistencia interna estimada mediante alfa de Cronbach arrojó valores adecuados (Ver apartado Resultados para conocer propiedades psicométricas de la versión adaptada).

- Escala de compromiso organizacional (Allen \& Meyer, 1990; Omar, 2005): Esta prueba está integrada por 18 ítems con formato Likert de cinco posiciones, con recorrido de 1 (totalmente en desacuerdo) a 5 (totalmente de acuerdo). Evalúa cada uno de los tres tipos de compromiso organizacional $(\mathrm{CO})$ a través de seis ítems cada uno. El compromiso afectivo (i. e., "Estaría feliz si pasara el resto de mi carrera en la empresa donde trabajo), el compromiso normativo (i. e., "Esta organización merece mi lealtad") y el compromiso calculativo (i.e., "Siento que tengo pocas opciones de trabajo como para dejar mi organización"). Los índices de consistencia interna (alfa de Cronbach) de la versión daptada a la Argentina (Omar, 2005) fueron de .82 para el compromiso afectivo, .73 para el normativo y .76 para el calculativo.

- Cuestionario de estrés laboral (OIT/OMS; en Llaneza Álvarez, 2009): Se trata de una prueba elaborada en conjunto por la Organización Internacional del Trabajo (OIT) y la Organización Mundial de la Salud (OMS) con el fin de evaluar el nivel de estrés percibido por los trabajadores. La evaluación se realiza en virtud de siete ejes que reflejan posibles estresores laborales (Clima Organizacional, Estructura Organizacional, Territorio Organizacional, Tecnología, Influencia del Líder, Falta de Cohesión, Respaldo del Grupo). Está integrada por 25 ítems con respuesta de formato Likert de siete puntos con un recorrido de 1 (indica que la condición descripta NUNCA es fuente de estrés) a 7 (indica que la condición descripta SIEMPRE es fuente de estrés), que marca con qué frecuencia la condición descripta es considerada una fuente actual de estrés (i.e. "La forma de rendir informes entre superior y subordinado me hace sentir presionado"). Según los autores, puntajes mayores a 153 indican un alto nivel de estrés. A los fines de este estudio se calculó alfa de Cronbach para la escala total obteniéndose un valor de .94 .

- Escala de satisfacción con la vida-SWLS(Diener, Emmons, Larsen, \& Griffin, 1985): Es una escala de cinco ítems que se responden en un formato Likert de 7 categorías que examinan el grado de satisfacción global con la vida (i.e. "En la mayoría de los sentidos, mi vida está cerca de mis ideales"). Se utiliza internacionalmente 
para evaluar el bienestar en tanto componente cognitivo de la satisfacción. Diferentes estudios empíricos han informado buenos índices de validez y fiabilidad (Diener et al., 1985). Se trabaja con una puntuación promedio que indica el grado de satisfacción percibida por el evaluado. En este caso se utilizó una versión adaptada al idioma español para su uso en población argentina (Castro Solano, 2000). La fiabilidad evaluada mediante el coeficiente alfa de Cronbach para la muestra de los participantes de este estudio fue de .85 .

Además, se diseñó una serie de protocolos para la evaluación de algunas de las variables consideradas en el estudio. Dichos protocolos fueron testeados previamente en un estudio piloto realizado con un grupo reducido de empleados $(n=15)$. Se modificaron algunas expresiones lingüísticas sugeridas por los participantes del estudio piloto dando lugar a las versiones definitivas utilizadas. A continuación, se describen los protocolos diseñados:

- Encuesta Sociodemográfica: Recaba datos personales de los participantes (sexo, edad, lugar de residencia, estado civil, nivel socioeconómico, nivel de estudios, ocupación, nivel socio - económico).

- Encuesta Organizacional: Consulta a los participantes sobre datos de la organización en la que trabajan y el puesto que ocupan (tamaño, tipo, rubro, área en la que trabaja, puesto y personal a cargo). Además, incluye dos ítems en los que se solicita que quienes responden listen tres características positivas y tres negativas que asocian a la organización (i.e. "Enumere tres características que Ud. considere positivas de la organización en la que trabaja"). Dichas características se analizaron en un estudio previo en el que se analizaron perfiles de organizaciones positivas (Lupano Perugini \& Castro Solano, 2016).

- Encuesta de Satisfacción Laboral: Se diseñaron seis ítems con opción Likert de respuesta que va de 1 (totalmente insatisfecho) a 7 (totalmente satisfecho) que intentan evaluar cuán satisfecha se autopercibe la persona en cuanto a su trabajo en general y diferentes aspectos del mismo (i.e., sueldo, jefes, compañeros, lugar, carrera). Un ejemplo de ítem es "¿Cuán satisfecho estoy con el sueldo que recibo?". Para la elección de las áreas a evaluar (e.g. sueldo, jefes, compañeros) se tomaron en cuenta aspectos analizados en otros instrumentos previos (e.g., Balzer et al., 1997). Se calculó alfa de Cronbach para la escala total obteniéndose un valor aceptable de .76

- Encuesta sobre Desempeño-performance Organizacional e Individual: Frente a la imposibilidad de obtener indicadores objetivos sobre el rendimiento organizacional se decidió inferirlo a partir de la percepción de los empleados. Se tomaron como indicadores de desempeño organizacional algunos utilizados por Cameron, Brigth, $\mathrm{y}$ Caza (2004) en sus investigaciones sobre virtudes y performance. Los mismos aluden a niveles de eficiencia, innovación, crecimiento, calidad, retención de empleados y clientes, satisfacción, adaptación. Por ejemplo, para el diseño de la primera sección de la encuesta -orientada a la evaluación del desempeño organizacional- se construyeron diez ítems con opción Likert de respuesta -1 (Poco) a 6 (Mucho)-, considerando los indicadores de eficiencia propuestos por Cameron et al. (2004) (e.g. cumplimento de objetivos, atención a las demandas del cliente, utilización óptima de recursos, etc). Un ejemplo de ítem es “¿En qué medida cree que la organización cumplió eficientemente -haciendo un buen uso de los recursos- con los objetivos propuestos?". El alfa obtenido para este apartado fue de .89. Siguiendo el mismo criterio, se diseñó otra sección que solicitaba al participante que calificara su desempeño como empleado. Se diseñaron seis ítems con la misma opción de respuesta que el apartado anterior $(1=$ Poco y $6=$ Mucho $)$. Un ejemplo de ítem es: "¿En qué medida cree que los resultados obtenidos fueron de calidad?". El alfa obtenido para esta sección fue de .84 .

\section{Procedimiento}

Los datos fueron recolectados por alumnos que se encontraban realizando una práctica de investigación en una universidad privada de la ciudad de Buenos Aires. Los participantes fueron voluntarios y no recibieron retribución alguna por su colaboración. Además, el cuadernillo que contenía las encuestas presentaba en su portada una introducción en la que se solicitaba el consentimiento del participante, se aseguraba el anonimato de los datos y su uso exclusivo para investigación. La recolección y carga de datos fue supervisada por un docente investigador.

Para la carga y análisis de los datos se utilizaron los programas SPPS 18.0 y EQS 6.2. 


\section{Resultados}

En primer lugar se llevó a cabo la validación de la escala EACT. A fin de poner a prueba la estructura factorial de dicha escala se calculó un análisis factorial confirmatorio en el que se ponía a prueba el modelo de tres factores - atención, absorción, energía-. Aunque las características de la distribución no parecían diferir demasiado de la distribución normal -ver tabla1-, se usó una matriz de correlaciones policóricas ya que resulta más apropiada cuando las opciones de respuesta se encuentran formuladas en formato Likert (Freiberg Hoffmann, Stover, de la Iglesia, \& Fernández Liporace, 2013; Muthén \& Kaplan, 1985). Para estudiar el ajuste del modelo de tres factores se evaluaron distintos índices: CFI (Comparative Fit Index), NFI (Normed Fit Index), IFI (Incremental Fit Index) y RMSEA (Root Mean Square Error of Approximation). Además, se valoraron los pesos de regresión de cada ítem en su correspondiente variable latente.

Tabla 1.

Estadísticos descriptivos de los ítems

\begin{tabular}{lccc}
\hline & M(DE) & Asímetría & Curtosis \\
\cline { 2 - 4 } Ítem 1 & $3.18(1.03)$ & -0.20 & -0.56 \\
Ítem 2 & $3.54(0.97)$ & -0.42 & -0.46 \\
Ítem 3 & $3.57(0.91)$ & -0.58 & 0.14 \\
Ítem 4 & $3.82(0.83)$ & -0.82 & 1.16 \\
Ítem 5 & $3.50(1.07)$ & -0.44 & -0.63 \\
Ítem 6 & $3.80(0.84)$ & -0.62 & 0.50 \\
Ítem 7 & $3.05(0.96)$ & 0.08 & -0.31 \\
Ítem 8 & $3.70(0.88)$ & -0.74 & 0.60 \\
Ítem 9 & $3.86(0.87)$ & -0.86 & 0.88 \\
Ítem 10 & $3.89(0.80)$ & -0.78 & 1.25 \\
Ítem 11 & $3.49(0.87)$ & -0.47 & 0.23 \\
Ítem 12 & $3.95(0.75)$ & -0.82 & 1.60 \\
\hline
\end{tabular}

Los resultados indicaron que el ítem 4 no mostraba un peso de regresión apropiado $(<.40)$ y por ello fue eliminado. El análisis se realizó otra vez sin el ítem 4 obteniéndose índices que mostraban un excelente ajuste del modelo: CFI $=.986$, $\mathrm{NFI}=.979, \mathrm{IFI}=.986, \mathrm{RMSEA}=.059(\mathrm{IC} 90 \%=$ $.046-.071)$. En la figura 1 puede observarse que todos los pesos de regresión de los ítems resultaron apropiados (Byrne, 2006; Kline, 2000).

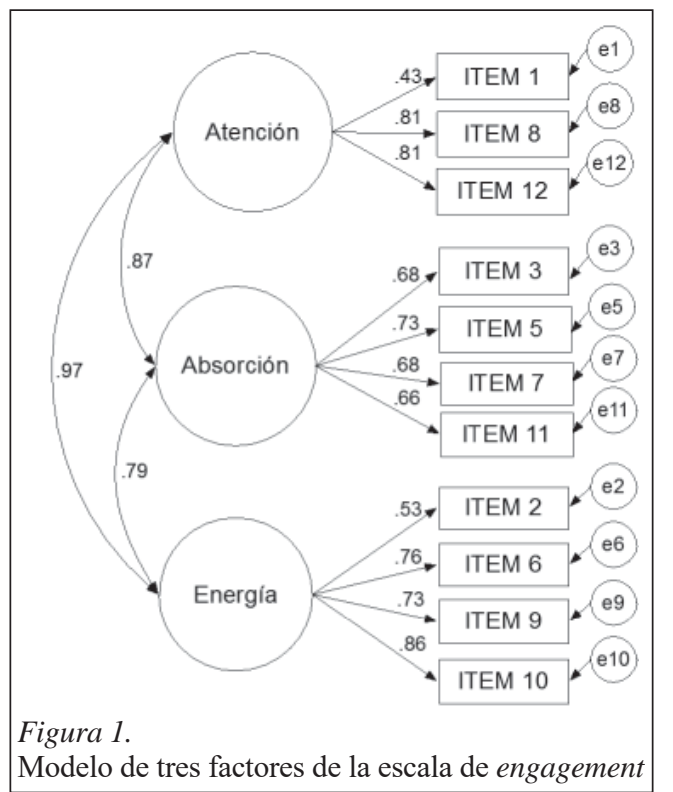

En virtud de las altas correlaciones halladas entre los factores se puso a prueba un modelo unidimensional. Para estudiarlo se realizó el mismo procedimiento que con el modelo de tres factores, hallándose índices de ajuste adecuados pero inferiores: $\mathrm{CFI}=.972, \mathrm{NFI}=.964, \mathrm{IFI}=.972$, RMSEA $=.078($ IC 90\% $=.067-.089)$. Además, los pesos de las regresiones fueron peores que en el modelo anterior (ver figura 2), confirmando que el modelo de tres factores resulta más adecuado.

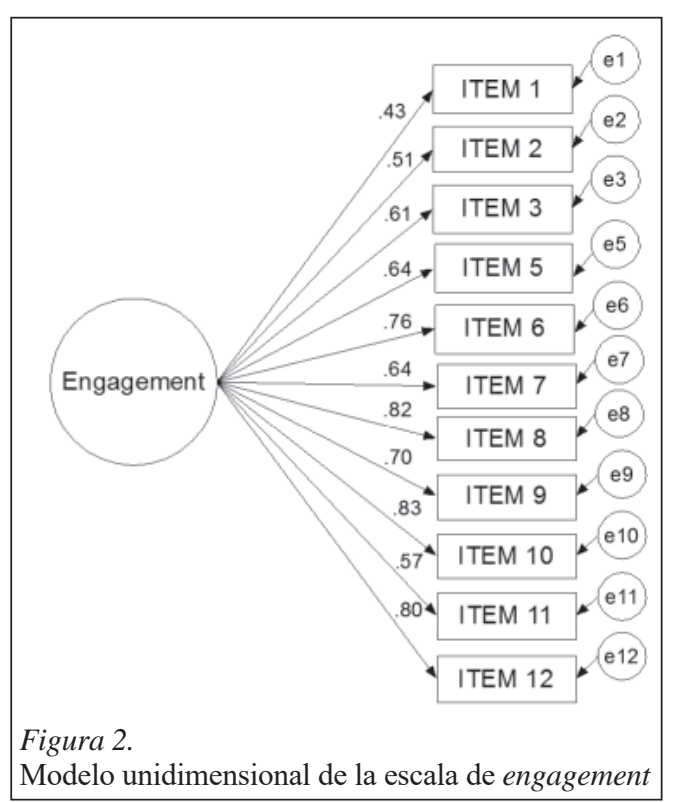


Con el fin de obtener evidencias de validez convergente y discriminante se correlacionaron las subescalas atención, absorción y energía con distintas medidas que se emplearon como criterios: (a) una medida de Satisfacción con la Vida; (b) tres medidas de Compromiso Organizacional -Afectivo, Calculativo y Normativo; y, (c) una medida de Estrés. En la tabla 2 se aprecia que en el caso de la medida de Satisfacción, las tres escalas correlacionaron de manera estadísticamente significativa, positiva y débil. Esto evidencia cierta convergencia entre los constructos evaluados y descarta la hipótesis de que se trate de constructos solapados. En cuanto a las medidas de Compromiso, las correlaciones indicaron similares resultados a excepción del Compromiso Afectivo, que correlacionó de forma moderada con Atención y Energía. Finalmente, se obtuvieron correlaciones estadísticamente significativas, negativas y muy débiles para las escalas Atención y Absorción con la medida de Estrés. La correlación entre Estrés y Energía no fue estadísticamente significativa $(p>.05)$.

Tabla 2.

Pruebas de validez convergente y discriminante

\begin{tabular}{lccc}
\hline & Atención & Absorción & Energía \\
\cline { 2 - 4 } $\begin{array}{l}\text { Satisfacción } \\
\text { con la Vida }\end{array}$ & $.249^{* *}$ & $.289^{* *}$ & $.243^{* *}$ \\
$\begin{array}{l}\text { Compromiso } \\
\text { Afectivo }\end{array}$ & $.434^{* *}$ & $.270^{* *}$ & $.376^{* *}$ \\
$\begin{array}{l}\text { Compromiso } \\
\text { Calculativo }\end{array}$ & $.157^{* *}$ & $.109^{* *}$ & $.183^{* *}$ \\
$\begin{array}{l}\text { Compromiso } \\
\text { Normativo }\end{array}$ & $.310^{* *}$ & $.238^{* *}$ & $.296^{* *}$ \\
Estrés & $-.101^{*}$ & $-.111^{* *}$ & -.062 \\
\hline${ }^{* *} p<.01$ & & & \\
${ }^{*} \mathrm{p}<.05$ & & & \\
& & &
\end{tabular}

Por último, para estimar la consistencia interna se calculó el coeficiente alfa de Cronbach para las tres escalas obteniéndose valores aceptables (Kline, 2000; Nunnally \& Berstein, 1995): Atención $=.70 ;$ Absorción $=.74 ;$ Energía $=.76$

Una vez validada la escala EACT, se pretendió estudiar si se verificaban diferencias en el nivel de engagement de acuerdo con variables individuales como sexo, edad y personal a cargo. En cuanto al sexo de los participantes, no se hallaron diferencias estadísticamente significativas $(p>.05)$.
Para estudiar diferencias de acuerdo con la edad se dividió la muestra en menores de 40 años $(n=380)$ y sujetos con 40 años o más $(n=186)$. Una prueba t de Student indicó que quienes tenían menos de 40 años presentaban mayor Atención $(t=-4.427 ; 564 \mathrm{gl} ; p<.001)$ y absorción $(t=-2.679$; $564 \mathrm{gl} ; p=.008)$. No se hallaron diferencias en la Energía según grupo de edad $(p>.05)$. Por otro lado, se encontró que quienes tenían personal a cargo mostraban menor nivel de Atención en comparación con quienes no tenían personal a cargo $(t=-5.095 ; 566 \mathrm{gl} ; p<.001)$. No se encontraron diferencias estadísticamente significativas en los niveles de Absorción y Energía entre quienes tenían personal a cargo y quienes no lo tenían $(p>.05)$.

También resultaba de interés analizar si los niveles de engagement variaban de acuerdo con variables organizacionales. Una prueba ANOVA one-way no verificó diferencias en los tres tipos de engagement según el tamaño de la empresa $(p>.05)$. Finalmente, tampoco se encontraron diferencias en el tipo de engagement entre quienes pertenecían a una empresa tipo pública y quienes trabajaban en una privada $(p>.05)$.

Por último, resultó de interés identificar distintos perfiles de engagement, obtenidos a partir de la escala validada en el presente estudio, asociados a alta performance y satisfacción laboral. Para ello se calculó un análisis de conglomerados jerárquico utilizando el método Ward y la distancia euclídea al cuadrado. Esto se hizo con el fin de determinar el número adecuado de clusters que maximizaba la diferencia entre los grupos y minimizaba las diferencias intragrupales. El historial del coeficiente de conglomeración y el dendograma indicaron que una solución de tres conglomerados era la más adecuada.

Se solicitaron tres clusters en el análisis de clusters de $\mathrm{k}$ medias. Los ANOVAs validaron la solución de tres clusters ya que indicaban diferencias estadísticamente significativas en los tres tipos de engagement de acuerdo con la pertenencia al cluster $(\mathrm{p}<.01)$. En la tabla 3 se consignan las medias para cada tipo de engagement según el cluster de pertenencia. Puede observarse que el cluster 1 podría denominarse "engagement bajo", el cluster 2 "engagement moderado" y el cluster 3 "engagement alto". 
Tabla 3.

Perfiles de engagement

\begin{tabular}{lccc}
\hline & Atención & Absorción & Energía \\
\cline { 2 - 4 } Cluster 1 & 2.60 & 2.54 & 2.81 \\
Cluster 2 & 3.58 & 3.32 & 3.75 \\
Cluster 3 & 4.34 & 4.14 & 4.45 \\
\hline
\end{tabular}

Se analizaron posibles diferencias en la Satisfacción Laboral y la Performance Organizacional y del empleado según el cluster de pertenencia. Los ANOVAs indicaron que diferencias estadísticamente significativas tanto para Satisfacción Labo$\operatorname{ral}(F=22.632 ; 2 \mathrm{gl} ; p<.001)$, como para Performance Organizacional $(F=39.359 ; 2 \mathrm{gl} ; p<.001)$ y Performance del Empleado ( $F=10.927 ; 2 \mathrm{gl} ; p<$ $.001)$. Las pruebas post-hoc Bonferroni indicaron que en todos los casos había diferencias estadísticamente significativas entre los tres clusters. Quienes pertenecían al cluster del "engagement alto" referían mayor Satisfacción Laboral, Performance Organizacional y del empleado en comparación con los otros dos clusters. Y quienes pertenecían al cluster de "engagement moderado" tenían mayor presencia de las tres variables en comparación con los de "engagement bajo" (ver tabla 4).

\section{Discusión}

El objetivo principal de este estudio consistió en la validación de una escala para la evaluación del work engagement basada en la propuesta teórica de Rothbard y Patil (2012). Según el análisis factorial confirmatorio efectuado, se ha verificado la estructura de tres factores tal como propone el modelo teórico señalado. Tal estructura de tres factores ha presentado, incluso, un mejor ajuste que una estructura unidimensional, puesto a prueba en virtud de las altas correlaciones halladas entre los factores.

Además, se verificó una buena consistencia interna y se obtuvieron evidencias de validez convergente y discriminante. Las correlaciones débiles y moderadas halladas entre las dimensiones de work engagement y Satisfacción Laboral, por un lado, y Compromiso Organizacional, por otro, dan cuenta de que las variables analizadas se encuentran relacionadas y descartan, además, la hipótesis de que pueda tratarse de constructos solapados tal como ha sido cuestionado por algunos autores (e.g. Macey \& Schneider, 2008). En relación con lo antedicho, Saks (2006) argumenta que el work engagement difiere de dichos constructos en tanto la Satisfacción y el Compromiso Organizacional son consideradas actitudes hacia la organización y el trabajo mientras que el engagement se entiende como una presencia en un rol, y no como una actitud. Además, el engagement suele ser resultado de la presencia de dichas actitudes. Es decir, las personas darían lo mejor de sí cuando se sienten comprometidas y satisfechas con su lugar de trabajo y tareas. Por otro lado, las correlaciones halladas con medidas de estrés darían cuenta de que el engagement se asocia negativamente con la presencia del mismo. Sin embargo, en virtud de que dichas correlaciones fueron débiles también descartaría la teoría de Maslach, Schaufeli y Leiter (2001) que entienden el work engagement como el polo opuesto al burnout. Estos resultados justificarían, entonces, la elección de la propuesta teórica escogida.

En la presente investigación también se buscó analizar posibles diferencias en los niveles de work engagement de acuerdo con algunas variables individuales y organizacionales. En general, los antecedentes que estudiaron la relación con va-

Tabla 4.

Diferencias en Satisfacción Laboral y la Performance Organizacional y del empleado de acuerdo con los perfiles de engagement

\begin{tabular}{lccc}
\hline & $\begin{array}{c}\text { Engagement } \\
\text { alto }\end{array}$ & $\begin{array}{c}\text { Engagement } \\
\text { moderado }\end{array}$ & $\begin{array}{c}\text { Engagement } \\
\text { bajo }\end{array}$ \\
\cline { 2 - 4 } Satisfacción Laboral & $5.22^{\mathrm{A}}$ & $4.85^{\mathrm{B}}$ & $4.32^{\mathrm{C}}$ \\
Performance Organizacional & $4.30^{\mathrm{A}}$ & $4.05^{\mathrm{B}}$ & $3.73^{\mathrm{C}}$ \\
Performance del Empleado & $4.95^{\mathrm{A}}$ & $4.61^{\mathrm{B}}$ & $4.03^{\mathrm{C}}$ \\
\hline Letras iguales indican subconjuntos homogéneos de acuerdo al análisis pos hoc (Bonferroni)
\end{tabular}


riables como la edad y el sexo suelen ser bastante contradictorios (Goštautaitė \& Bučiūnienè, 2015). En la muestra analizada no se hallaron diferencias en cuanto al sexo aunque estudios previos reportaron leves diferencias a favor de los hombres (e.g. Liu, Cho, \& Putra, 2017; Mastenbroek et al., 2014). Por otro lado, se observaron mayores niveles de Atención y Absorción en sujetos de menor edad. Estos resultados estarían en sintonía con los estudios que mostrarían un incremento del engagement hasta cierto nivel de edad (e.g. James, McKechnie, \& Swanberg, 2011). No se encontraron diferencias en cuanto al tamaño y tipo de empresa abonando la hipótesis de Innstrand (2016), que sostiene que el tipo de tarea tendría más influencia con el compromiso que se establece con ella que con variables individuales o asociadas al tipo de organización. Según estos resultados sería interesante analizar en futuros estudios los niveles de engagement según el tipo de tarea (e.g. atención al público, docente, etc.).

El último objetivo de este trabajo apuntaba a identificar perfiles de engagement asociados a alta Performance y Satisfacción Laboral. Los análisis efectuados mostraron que los empleados que pertenecían al cluster del "engagement alto" manifestaban mayor Satisfacción Laboral, Performance Organizacional y del Empleado. Estos resultados confirman que el engagement se encuentra asociado a altos niveles de Satisfacción y Performance tal como sostienen los antecedentes consultados (e.g. Alarcon \& Edwards, 2011; Christian et al., 2011; Høigaard et al., 2012; Mache et al., 2014; Karatepe \& Karadas, 2015). Un empleado con alto engagement estaría más motivado para llevar adelante las tareas de su rol con mayores persistencia, intensidad y concentración y eso, en general, deriva en mejores resultados y satisfacción.

\section{Conclusiones}

Los resultados expuestos confirman que la EACT es una prueba con adecuadas evidencias de validez interna y externa, a la vez que confiable para le medición del work engagement en población argentina. Por otra parte, el interés de los resultados obtenidos para el área aplicada se aprecia de modo inmediato. Las áreas de Personal o Recursos Humanos de las organizaciones podrían beneficiarse, entonces, reforzando los niveles de engagement de los empleados ya que aquí se ha verificado su influencia sobre el rendimiento.
Algunas limitaciones del estudio realizado se relacionan con el empleo de medidas subjetivas para estimar el desempeño individual y organizacional. Ellas podrían complementarse con nuevos desarrollos de medidas complementarias. Asimismo, la muestra analizada debiera balancearse en cuanto al porcentaje de representación de empresas públicas y privadas según sus porcentuales en la población organizacional local.

Finalmente, futuros estudios deberían propiciar el análisis de modelos multivariados que reúnan tanto variables de nivel individual -como puede ser el work engagement, capital psicológico, compromiso organizacional, entre otras-, como variables de nivel organizacional. Por ejemplo, estudios previos han mostrado que la percepción de virtudes en el nivel organizacional (e.g. apoyo y respeto, perdón, inspiración) tiene influencia sobre variables de resultado en los niveles individual y organizacional (e.g. Cameron, Mora, Leutscher, \& Calarco, 2011; Lupano Perugini \& Castro Solano, 2017). El diseño de modelos multivariados permitirá, así, identificar los mejores predictores - personales y organizacionales -de rendimiento y satisfacción laboral.

\section{Referencias}

Alarcon, G. \& Edwards, J. (2011). The Relationship of Engagement, Job Satisfaction and Turnover Intentions. Stress and Health Volume, 27(3), 294-298. https://doi. org/10.1002/smi.1365

Albrecht, S.L., Bakker, A.B., Gruman, J.A., Macey, W.H. \& Saks, A.M. (2015) Employee engagement, human resource management practices and competitive advantage: An integrated approach. Journal of Organizational Effectiveness: People and Performance, 2(1), 7-35. https://doi.org/10.1108/ joepp-08-2014-0042

Allen, N. J. \& Meyer, J. P. (1990). The Measurement and Antecedents of Affective, Continuance and Normative Commitment to the Organization. Journal of Occupational Psychology, 63, 1-18. https://doi. org/10.1111/j.2044-8325.1990.tb00506.x

Avery, D. R., McKay, P. F., \& Wilson, D. C. (2007). Engaging the aging workforce: The relationship between perceived age similarity, satisfaction with coworkers, and employee engagement. Journal of Applied Psychology, 92(6), 1542-1556. https://doi. org/10.1037/0021-9010.92.6.1542

Bakker, A. B., Schaufeli, W. B. \& Leiter, M. P. (2008). Work engagement: An emerging concept in occupational health psychology. Work \& Stress, 22(3), 187-200. https://doi.org/10.1080/02678370802393649

Balzer, W. K., Kihm, J. A., Smith, P. C., Irwin, J. L., Bachiochi, P. D., Robie, C., Sinar, E. F., \& Parra, L. F. (1997). User's manual for the Job Descriptive Index (JDI; 1997 Revision) and the Job in General (JIG) Scales. Bowling Green, OH: Bowling Green State University. 
Byrne, B.M. (2006). Structural Equation Modeling With EQS: Basic Concepts, Applications, and Programming, Second Edition. New York: Psychology Press. https:// doi.org/10.1207/s15328007sem1302_7

Castro Solano, A. (2000). Estilos de personalidad, objetivos de vida y satisfacción vital. Un estudio comparativo con adolescentes argentinos. (Tesis Doctoral inédita). Facultad de Psicología. Universidad Complutense de Madrid, España.

Cameron, K. S. \& Spreitzer, G. M. (2012). The Oxford Handbook of Positive Organizational Scholarship. New York: Oxford University Press.

Cameron, K.S., Brigth, D. \& Caza, A. (2004). Exploring the relationship between organizational virtuosness and performance. American Behavioral Scientist, 47(6), 1-24. https://doi.org/10.1177/0002764203260209

Cameron, K.S., Mora, C., Leutscher, T. \& Calarco, M. (2011). Effects of positive practices on organizational effectiveness. The Journal of applied Behavioral Science, 47, 266-308. https://doi.org/10.1177/0021886310395514

Christian, M. S., Garza, A. S. \& Slaughter, J. E. (2011). Work Engagement: A Quantitative Review and Test of its Relations with Task and Contextual Performance. Personnel Psychology, 64, 89-136. https://doi. org/10.1111/j.1744-6570.2010.01203.x

Diener, E., Emmons, R. A., Larsen, R. J., \& Griffin, S. (1985). The satisfaction with life scale. Journal of Personality Assessment, 49, 71-75. https://doi.org/10.1207/ s15327752jpa4901_13

Freiberg-Hoffmann, A., Stover, J. B., de la Iglesia, G., \& Fernández-Liporace, M. (2013). Correlaciones policóricas y tetracóricas en estudios factoriales exploratorios y confirmatorios. Ciencias Psicológicas, 7(2), 151-164. https://doi.org/10.22235/cp.v7i1

Goštautaitè, B. \& Bučiūnienè, I. (2015). Work engagement during lifespan: the role of interaction outside the organization and task significance. Journal of Vocational Behavior, 89, 1 9-119. https://doi.org/10.1016/j. jvb.2015.05.001

Hirschfeld, R.R. \& Thomas, C.H. (2008). Representations of trait engagement: Integration, additions, and mechanisms. Industrial and Organizational Psychology, 1, 6366. https://doi.org/10.1111/j.1754-9434.2007.00011.x

Høigaard, R., Giske, R. \& Sundsli, K. (2012). European Journal of Teacher Education, 35 (3), 347-357. https:// doi.org/10.1080/02619768.2011.633993

Innstrand, S.T. (2016). Occupational Differences in Work Engagement: A Longitudinal Study Among Eight Occupational Groups in Norway. Scandinavian Journal of Psychology, 57(4), 338-349. https://doi.org/10.1111/ sjop. 12298

James, J. B., McKechnie, S., \& Swanberg, J. (2011). Predicting Employee Engagement in an AgeDiverse Retail Workforce. Journal of Organizational Behavior, 32(2), 173-196. https://doi.org/10.1002/job.681

Jeung, C.W. (2011). The Concept of Employee Engagement: A Comprehensive Review from a Positive Organizational Behavior Perspective. Performance Improvement Quarterly, 24, 2, 49-69. DOI: 10.1002/piq.

Kahn, W. A. (1990). 'Psychological conditions of personal engagement and disengagement at work'. Academy of Management Journal, 33, 692-724. https://doi. org/10.2307/256287

Kahn, W.A. (1992). 'To be fully there: psychological presence at work'. Human Relations, 45(4), 321-349. https://doi. org/10.1177/001872679204500402
Karatepe, O.M. \& Karadas, G. (2015). Do psychological capital and work engagement foster frontline employees' satisfaction?: A study in the hotel industry. International Journal of Contemporary Hospitality Management, 27 (6),1254-1278. https://doi. org/10.1108/IJCHM-01-2014-0028

Kline, P. (2000). Handbook of psychological testing. London: Routledge.

Liu, J., Cho, S. \& Putra, E. D. (2017). The moderating effect of self-efficacy and gender on work engagement for restaurant employees in the United States. International Journal of Contemporary Hospitality Management, 29(1), 624-642. https://doi.org/10.1108/IJCHM-10-2015-0539

Lin, W., Wang, L., Bamberger, P. A., Zhang, Q., Wang, H., Guo, W., Shi, J., \& Zhang, T. (2016). Leading future orientations for current effectiveness: The role of engagement and supervisor coaching in linking future work self salience to job performance. Journal of Vocational Behavior, 92, 145-156. http://dx.doi. org/10.1016/j.jvb.2015.12.002

Llaneza Álvarez, F. J. (2009). Ergonomía y Psicosociología Aplicada. Manual para la formación del especialista, 12ed. Valladolid: Lex Nova

Lupano Perugini, M.L. \& Castro Solano, A. (2016). Perfiles de organizaciones positivas. Análisis de características percibidas según variables individuales, organizacionales y de resultado. Escritos de Psicología, 9(2), 1-11. https://doi.org/10.5231/psy.writ.2016.1103

Lupano Perugini, M.L. \& Castro Solano, A. (2017). Influencia de las Virtudes Organizacionales sobre la performance laboral. Un estudio en organizaciones argentinas. Interdisciplinaria, en prensa.

Lupano Perugini, M.L. (2014). Organizaciones Positivas: un marco de referencia para su abordaje en Latinoamérica. Acta Psiquiátr. Psicol. Am. Lat., 60(4), 277-284.

Macey, W.H. \& Schneider, B. (2008). The meaning of engagement. Industrial and Organizational Psychology, 1, 3-30. https://doi.org/10.1111/j.1754-9434.2007.0002.x

Mache S., Vitzthum K., Klapp B.F., \& Danzer, G. (2014) Surgeons' work engagement: influencing factors and relations to job and life satisfaction. Surgeon, 12(4), 181-190. doi: 10.1016/j. surge.2013.11.015

Maslach, C., Schaufeli, W. B. \& Leiter, M. P. (2001). 'Job burnout'. Annual Review of Psychology, 52, 397-422. https://doi.org/10.1146/annurev.psych.52.1.397

Masson, R.C., Royal, M.A., Agnew, T.G. \& Fine, S. (2008). Leveraging employee engagement: The practical implications. Industrial and Organizational Psychology, 1, 56-59. https://doi.org/10.1111/j.17549434.2007.00009.x

Mastenbroek, N. J. J. M., Jaarsma, A. D. C., Demerouti, E., Muijtjens, A. M. M., Scherpbier, A. J. J. A. \& van Beukelen, P. (2014). Burnout and engagement, and its predictors in young veterinary professionals: the influence of gender. Veterinary Record, 174(6), 144-152. https://doi.org/10.1136/vr.101762

Muthén, B., \& Kaplan D. (1985). A comparison of some methodologies for the factor analysis of non-normal Likert variables. British Journal of Mathematical and Statistical Psychology, 38, 171-189. https://doi. org/10.1111/j.2044-8317.1985.tb00832.x

Nienaber, H. \& Martins, N. (2014). An Employee Engagement Instrument and Framework Building on Existing Research. Mediterranean Journal of Social Sciences, 5(20), 485-496. https://doi.org/10.5901/mjss.2014. v5n20p485 
Nunnally, J., \& Berstein, I. (1995). Psychometric theory. New York: Mcgraw-Hill.

Omar, A. (2005). La cultura organizacional de las empresas argentinas [The organizational culture of Argentine companies]. Rosario: Conicet.

Owens, B.P., Baker, W.E., Sumpter, D.M., \& Cameron, K.S. (2016). Relational Energy at Work: Implications for Job Engagement and Job Performance. Journal of Applied Psychology, 101(1), 35-49. https://doi.org/10.1037/ ap10000032

Rich, B. L., LePine, J. A. \& Crawford, E. R. (2010). 7. Job Engagement: Antecedents and Effects On Job Performance. Academy of Management Journal, 53 (3), 617-635. https://doi.org/10.5465/AMJ.2010.51468988

Robertson, I.T. \& Cooper, C.L. (2010). Full engagement: The integration of employee engagement and psychological well-being. Leadership and Organizational Development, 31(4), 324-336. https://doi. org/10.1108/01437731011043348

Rodríguez-Montalbán, R., Martínez-Lugo, M., SánchezCardona, I. (2014). Análisis de las propiedades psicométricas de la Utrecht Work Engagement Scale en una muestra de trabajadores en Puerto Rico. Universitas Psychologica, 13(4), 1255-1266. https://doi. org/10.11144/Javeriana.UPSY13-4.appu

Rothbard, N. P. (2001). 'Enriching or depleting? The dynamics of engagement in work and family roles'. Administrative Science Quarterly, 46, 655-84. https:// doi.org/10.2307/3094827

Rothbard, N. P., \& Patil, S. V. (2012). Being There: Work Engagement and Positive Organizational Psychology. En K. S. Cameron \& G. M. Spreitzer (Eds.), The Oxford Handbook of Positive Organizational Scholarship (231243). New York: Oxford University Press.
Rothmann, S. \& Rothmann, S. (2010). Factors associated with employee engagement in South Africa. South African Journal of Industrial Psychology, 36(2), 925-937. https://doi.org/10.4102/sajip.v36i2.925

Saks, A.M. (2006). Antecedents and consequences of employee engagement. Journal of Managerial Psychology, 21(6), 600-619. https://doi.org/10.1108/02683940610690169

Schaufeli, W. B., \& Bakker, A. B. (2004). Job demands, job resources, and their relationship with burnout and engagement: A multi-sample study. Journal of Organizational Behavior, 25, 293-315. https://doi.org/10.1002/job.248

Schaufeli, W.B., Salanova, M., González-Romá, V. \& Bakker, A. B. (2002). The measurement of engagement and burnout and: A confirmative analytic approach. Journal of Happiness Studies, 3, 71-92. https://doi. org/10.1023/A:1015630930326

Spector, P.E. (1997). Job satisfaction: Application, assessment, causes and consequences. Thousand Oaks, CA: Sage.

Viljevac, A., Cooper-Thomas, H.D. \& Saks, A. (2012). An investigation into the validity of two measures of work engagement. The International Journal of Human Resource Management, 23, 17, 3692-3709. https://doi.or $\mathrm{g} / 10.1080 / 09585192.2011 .639542$

Zhang, Y., Rich, B. L. \& LePine, J.A. (2009). Transformational leadership and job performance: The mediating role of job engagement. Presented in an interactive paper session at the annual meeting of the Academy of Management. Chicago, August 8-11. 